\title{
Design and Simulation of On-Chip Lossy Transmission Line Pairs
}

\author{
Thomas Demeester ${ }^{*}$ and Daniël De Zutter (Fellow, IEEE) \\ Ghent University, Dept. of Information Technology \\ Sint-Pietersnieuwstraat 41, B-9000 Gent, Belgium \\ thomas.demeester@intec.ugent.be
}

\begin{abstract}
A quasi-TM reciprocity based multi-conductor transmission line model is used to investigate the influence of the geometry on the performance of on-chip transmission line pairs for high-frequency differential signal transmission. It is shown that both the knowledge of the fundamental transmission line modes and of the internal impedance of both connected circuits, are essential for a good design.
\end{abstract}

\section{Introduction}

In the design of very large scale integration (VLSI) circuits, wave phenomena on interconnects are becoming responsible for significant signal integrity problems, due to the always increasing frequencies. Commonly used at the highest frequencies is the technique of differential signaling in order to reduce the impact of electromagnetic noise and coupling with neighboring circuits. This means long interconnects have to be modeled as multi-conductor transmission lines. Only few electromagnetic models for the determination of the fundamental propagation modes allow the combined simulation of the finite conductivity of both the signal lines and the semiconducting substrate. An accurate quasi-TM twodimensional model has been proposed in [1], for the case of a single conductor. Based on power considerations, the current in the transmission line equivalent was proven to be a suitably weighted combination of the current in the signal line and the current through the substrate. The theory of [1] can be extended to the multi-conductor case, provided mode reciprocity is invoked instead of the conservation of power. A rigorous derivation of such a multi-conductor model within the quasi-TM frequency range is given in [2]. The use of the Dirichlet-Neumann boundary operator leads to a pure boundary integral equation formulation, allowing the fast simulation of complex configurations.

In this paper we propose some design criteria, based on the transmission line properties of differential lines in combination with the circuit impedances of source and load. The propagation characteristics of the lines will be determined by using the model from [2]. Further details regarding this model will be given in the oral presentation.

\section{Design of a differential pair}

Consider the two-dimensional structure shown in Fig. 1, with a conductor pair $\left(c_{1}, c_{2}\right)$ embedded in a dielectric layer on a semiconducting substrate, above a perfect electric conducting (PEC) ground. PEC walls are used to simulate a wide slab. A reference conductor $\left(c_{R}\right)$, kept on zero volt, and some neighboring conductors $\left(c_{3}-c_{5}\right)$ are also shown on Fig. 1 . The signal pair $\left(c_{1}, c_{2}\right)$ is e.g. needed to transmit high-frequency signals from a chip input amplifier to the output buffer. For a fixed line length of $960 \mu \mathrm{m}$, we will optimize the geometry parameters $\left(w_{S}, t, h, w_{R}, d\right)$ in order to minimize wave effects.

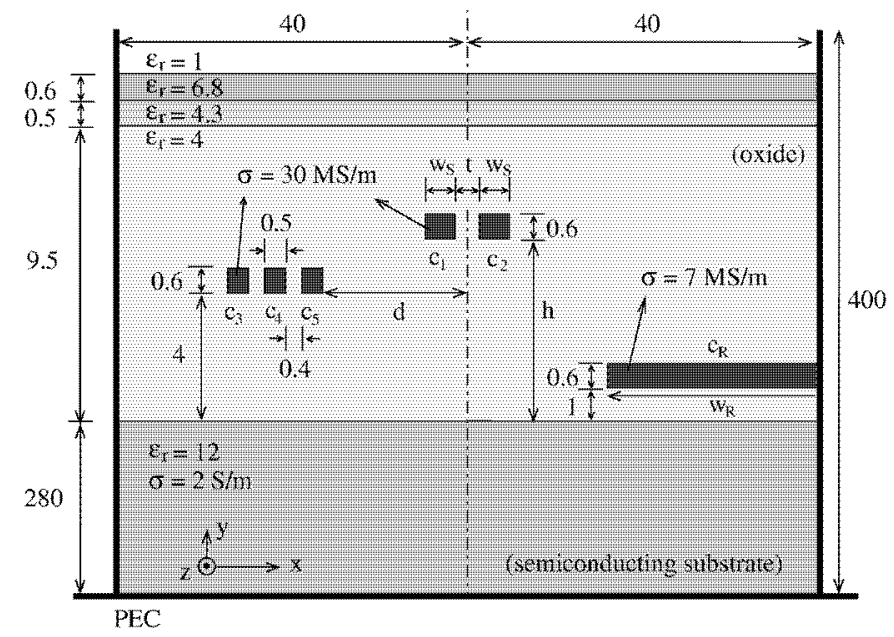

Fig. 1. On-chip transmission line structure with signal conductor pair $(\mathbf{c 1 , c 2})$. All dimensions are in micrometers.

The quasi-TM modal analysis of the signal lines leads to the capacitance and conductance matrices (per unit length) $C$ and $\boldsymbol{G}$, and the inductance and resistance matrices $\boldsymbol{L}$ and $\boldsymbol{R}$. The lines $c_{3}, c_{4}$ and $c_{5}$ are e.g. used to feed certain circuits with current. Here, we force their current to zero (which is a good approximation for high input impedance circuits), such that the $5 \times 5$ circuit matrices can be reduced to $2 \times 2$ matrices, relating voltages and currents on the signal lines only w.r.t. the reference conductor $c_{R}$ (except for Fig. 2, when the influence of line $c_{5}$ is investigated). The propagation constants are then found as the square root of the eigenvalues of $-(j \omega \boldsymbol{C}+\boldsymbol{G})(j \omega \boldsymbol{L}+\boldsymbol{R})$. For a perfectly symmetric configuration, the circuit matrices would have equal diagonal entries (subscript $s$ ) and equal off-diagonal entries (subscript $m$ ). The differential mode propagation constant $\beta_{d}$, corresponding to a $\left(e^{-j \beta_{d} z+j \omega x}\right)$ field dependence, would be given by

$\beta_{d}^{2}=-\left(j \omega\left(C_{s}+\left|C_{m}\right|\right)+\left(G_{s}-G_{m}\right)\right) \cdot\left(j \omega\left(L_{s}-L_{m}\right)+\left(R_{s}-R_{m}\right)\right)$

The configuration of Fig. 1 is not perfectly symmetric with respect to the dashed line in Fig. 1, but still the actual propagation constants agree with an almost entirely 'odd', resp. an 'even' mode. Hence, (1) can be used to explain the

\footnotetext{
${ }^{*}$ T. Demeester is a Research Fellow of the

Fund for Scientific Research, Flanders (FWO-V).
} 


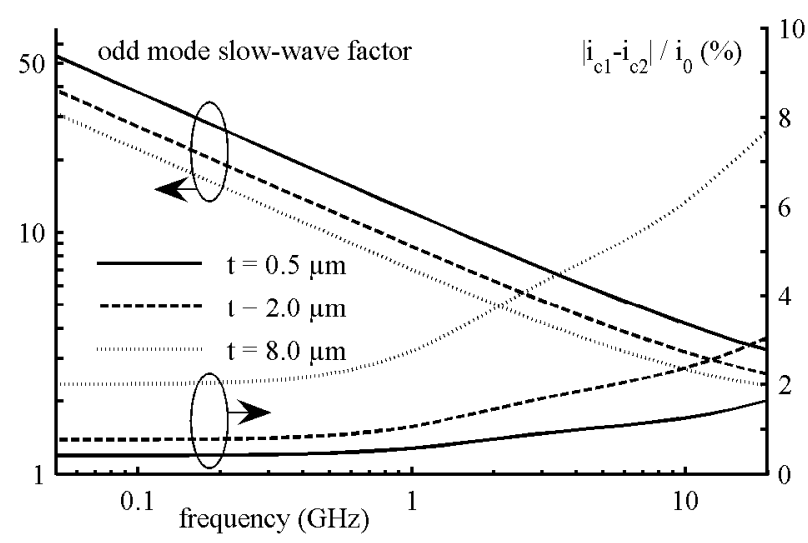

Fig. 2. Left axis: odd mode $S W F$, for $w_{S}=0.6, h=5.5$, $w_{R}=16, d=19.2$ (all in $\mu \mathrm{m}$ ), and for different values of $t$. Right axis: normalized differential signal current $\left|\mathbf{i}_{c 1}-\mathbf{i}_{\mathrm{c} 2}\right| \mathbf{i}_{\mathbf{0}}$, induced by a current $i_{0}$ through conductor $c_{5}$.

line properties for a differential excitation. On the one hand, we want to minimize the odd mode slow-wave factor (SWF), determined as $\operatorname{Re}\left(\beta_{d}\right) / k_{0}$ (with $k_{0}$ the free space wave number), and the attenuation $-\operatorname{Im}\left(\beta_{d}\right)$. The line then becomes electrically shorter (as the modal wave length is inversely proportional to the SWF), and wave effects are reduced. On the other hand, the differential characteristic impedance $Z_{\text {diff }}$ of the line,

$$
Z_{\text {diff }}=2 \sqrt{\left(j \omega\left(L_{s}-L_{m}\right)+\left(R_{s}-R_{m}\right)\right) /\left(j \omega\left(C_{s}+\left|C_{m}\right|\right)+\left(G_{s}-G_{m}\right)\right)}
$$

should remain large enough, to prevent the input impedance seen by the source from becoming too small, which in turn would lead to an unacceptable voltage drop. In the following, we will briefly discuss our results, but detailed physical explanations of the observed data will be presented orally.

Both the attenuation and the SWF decrease by increasing the distance $t$ between both signal conductors (see Fig. 2 for the SWF, the attenuation is not shown). However, $t$ has to remain much smaller than the distance $d$ to the nearest neighboring circuits or lines. As an illustration, we excite $c_{5}$ with a current $i_{0}$, while keeping $c_{1}$ and $c_{2}$ on zero volt. Fig. 2 shows the absolute value of the induced differential current component $\left(\mathrm{i}_{\mathrm{c} 1}-\mathrm{i}_{\mathrm{c} 2}\right)$ through the signal conductors. An increase in $t$ results in a higher induced current. Obviously, the effect is weaker for larger values of $d$.

Increasing the width $w_{S}$ of the signal conductors leads to a lower SWF and a lower attenuation. However, the SWF does not change much further, as soon as $R_{s}$ becomes lower than $j \omega L_{s}$ for the highest relevant frequencies (as is the case for $w_{S}=8.0 \mu \mathrm{m}$ in Fig. 3). Further widening the conductors only leads to a lower $Z_{\text {diff, }}$ as shown in Fig. 3. Fig. 4 shows how the signal lines are used in a source-load configuration. For typical but simplified (frequency-independent) impedances, the complex differential load voltage $V_{d}$ is shown, with the frequency as a parameter. For $w_{S}=0.5 \mu \mathrm{m}$, the SWF and attenuation are higher than for $w_{S}=2 \mu \mathrm{m}$, due to the larger line resistance. For $w_{S}=8 \mu \mathrm{m}, V_{d}$ is again lower, due to the decreased line impedance.

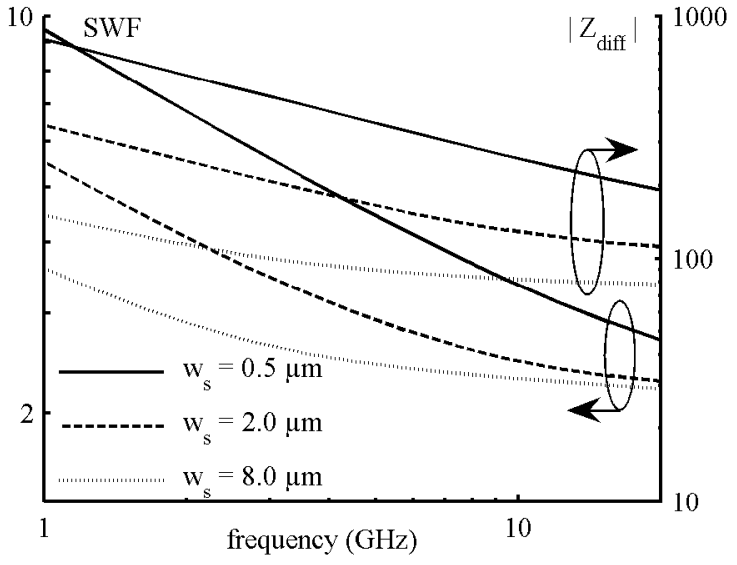

Fig. 3. Left axis: odd mode SWF, for $h=5.5, w_{R}=16$, $d=19.2$ and $t=2$ (all in $\mu \mathrm{m}$ ), and for different values of $w_{S}$. Right axis: diff. characteristic line impedance $\left|Z_{\text {diff }}\right|$ -

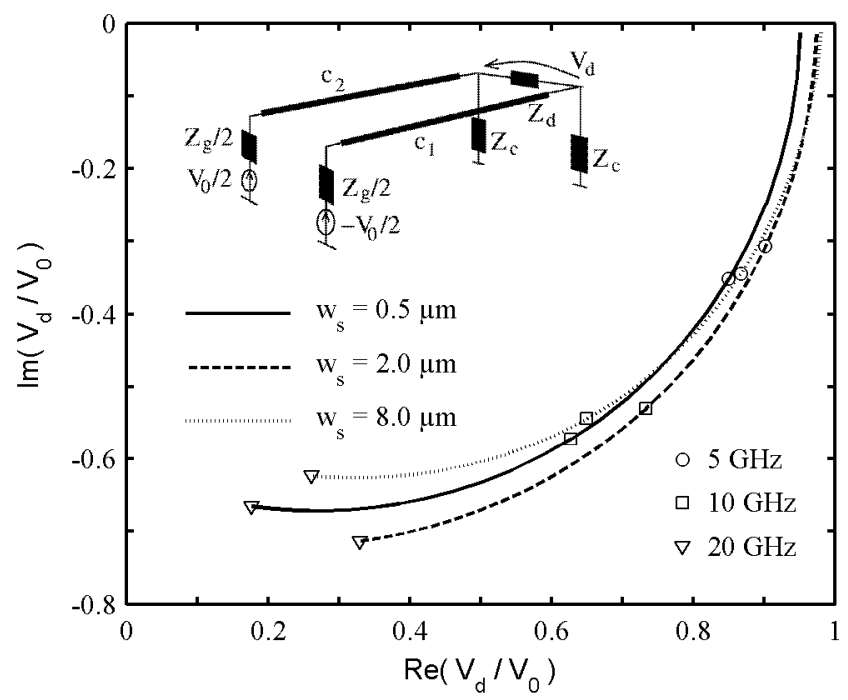

Fig. 4. Load voltage $V_{d}$ (for the same geometry as Fig. 3). Impedance values are $Z_{g}=120 \Omega, Z_{d}=Z_{c}=10 \mathrm{k} \Omega$.

Analogous considerations regarding the SWF and $Z_{\text {diff }}$, show that it is not recommended to increase $w_{R}$ such that $c_{1}$ and $\mathrm{c}_{2}$ become situated directly above the reference conductor, and that the height $h$ of the signal lines above the semiconducting substrate should be as large as possible.

\section{Conclusions}

It is shown how the performance of an on-chip differential pair interconnect depends on its geometry, considering both the transmission line properties and the circuits, connected by the lines.

\section{References}

[1] Plaza, G. et al, "Quasi-TM MoL/MoM approach for computing the transmission-line parameters of lossy lines," IEEE Trans. Microwave Theory Tech., Vol. 54, No. 1 (2006), pp. 198-209.

[2] Demeester, T. et al, "Quasi-TM transmission line parameters of coupled lossy lines based on the Dirichlet to Neumann boundary operator," IEEE Trans. Microwave Theory Tech., submitted for publication. 


\section{WORKSHOP PROGRAM}

$12^{\text {th }}$ IEEE Workshop on

\section{SIGNAL PROPAGATION ON INTERCONNECTS}

May 12-15, 2008 Popes' Palace, Avignon, France

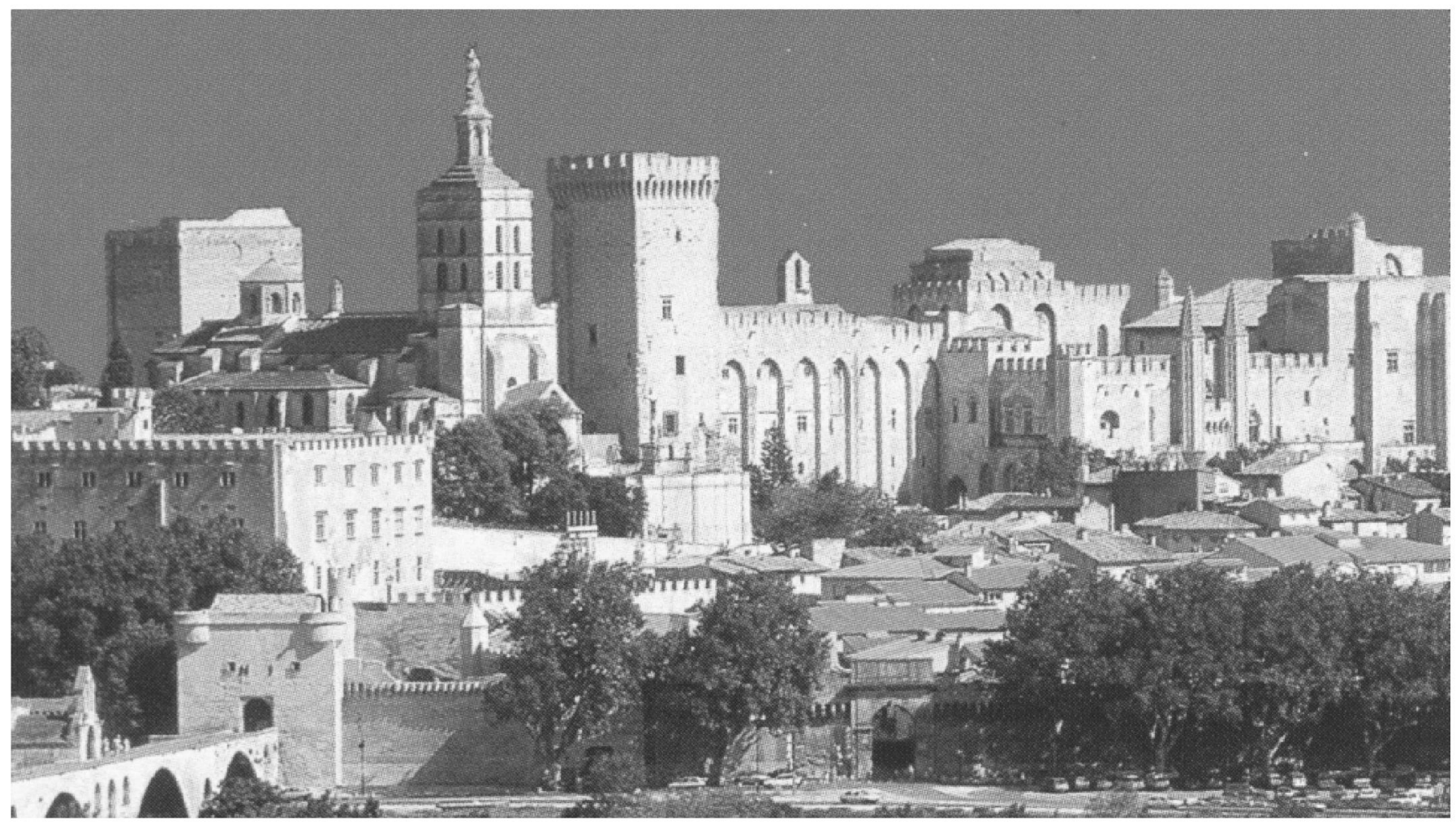

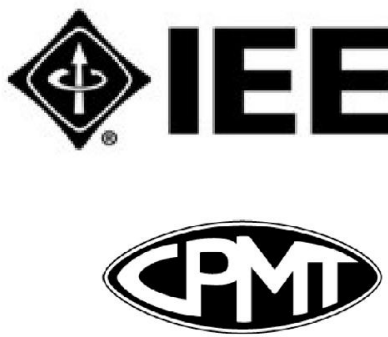

IEEE COMPONENTS, PACKAGING AND MANUFACTURING TECHNOLOGY SOCIETY

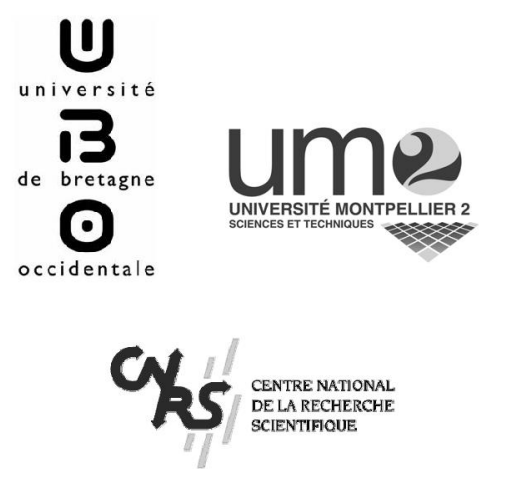

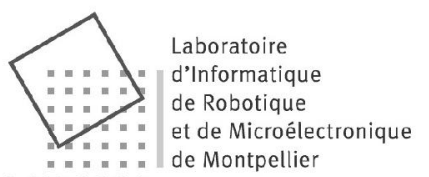

LIRMM

\section{Lab-STICC}

FRE CNRS 3167

Laboratoire en Sciences et

Technologie de l'Information, de la

Communication et de la

Connaissance 